\title{
Le eosinofilie polmonari. Descrizione di un caso clinico di polmonite eosinofila acuta da sertralina e revisione della letteratura
}

\section{Eosinophilic pneumonias. A clinical case of acute eosinophilic pneumonia associated with sertraline and literature review}

\section{Roberto Serini ${ }^{\text {a,* }}$, Rossano Dallari ${ }^{\text {a }}$, Ercole Turrini ${ }^{\text {a }}$, Alberto Debbi ${ }^{a}$, Chiara Benatti ${ }^{\mathrm{b}}$, Marco Grandi ${ }^{\mathrm{b}}$}

\author{
a UOC Pneumologia (Direttore: dott. Rossano Dallari), Nuovo Ospedale Civile di Sassuolo, AUSL di Modena
}

${ }^{\mathrm{b}}$ UOC Medicina Interna (Direttore: dott. Marco Grandi), Nuovo Ospedale Civile di Sassuolo, AUSL di Modena

Ricevuto il 3 luglio 2008; accettato il 14 novembre 2008

disponibile online il 13 luglio 2009

\section{KEYWORDS \\ Eosinophilic pneumonia; Pulmonary infiltrates; Pulmonary eosinophilia.}

\begin{abstract}
Summary
Background: Acute eosinophilic pneumonia (AEP) is one of the heterogeneous group of disorders termed eosinophilic lung diseases showing an abnormal accumulation of eosinophils in distal airways, air spaces, and the interstitial compartment of the lung. AEP is characterized by acute febrile respiratory failure, a typical radiographic pattern similar to that of acute pulmonary edema, eosinophilia in bronchoalveolar lavage (BAL), a dramatic response to corticosteroid therapy with no relapse when tapering or withdrawing treatment in the absence of infection. It can be idiopathic o secondary to known causes such as drugs, or fungal and parasitic infections. Clinical case: We report a case of a 76-year-old woman who developed symptoms, respiratory signs, and radiological and histological findings compatible with secondary AEP, we assessed as being associated with sertraline assumption (second case in literature).

Discussion: The AEP we detected could be related to a secondary hypersensitivity syndrome to drug exposure (DRESS syndrome: Drug Rush with Eosinophilia and Systemic Symptoms) characterized by 1 ) skin rush, 2) increased eosinophilia, and 3) systemic involvement. The presence of all three criteria confirmed our diagnosis.
\end{abstract}

(c) 2009 Elsevier Srl. All rights reserved.

\footnotetext{
* Corrispondenza: UOC Pneumologia, Dipartimento di Medicina, Nuovo Ospedale Civile di Sassuolo, via F. Ruini 2 - 41039 Sassuolo, MO. E-mail: robi.s@tin.it (R. Serini).
} 


\section{Introduzione}

Si definisce eosinofilia un abnorme accumulo di eosinofili nel sangue $\left(>350 / \mathrm{mm}^{3}\right)$ e/o nei tessuti.

L'eosinofilia viene arbitrariamente classificata in:

- lieve, quando gli eosinofili sono $>0,351 \times 10^{9} / \mathrm{L}$ e $<1,5$ $\times 10^{9} / \mathrm{L}$;

- moderata, quando gli eosinofili sono $>1,5 \times 10^{9} / \mathrm{L}$ e $<5$ $\times 10^{9} / \mathrm{L}$;

- severa, quando gli eosinofili sono $>5 \times 10^{9} / \mathrm{L}$ [1].

Tale condizione è il risultato di quattro processi:

- differenziazione e proliferazione degli eosinofili a livello midollare;

- interazione tra eosinofili e cellule endoteliali (adesione, migrazione);

- chemoattrazione e attivazione delle cellule;

- rapporto sopravvivenza/apoptosi.

L'eosinofilia può essere primitiva o secondaria ad altre patologie sottostanti. Nello specifico, la causa più comune è rappresentata dalle infezioni parassitarie, mentre nei Paesi industrializzati troviamo al primo posto le patologie allergiche, soprattutto da farmaci e atopiche.

Associate a eosinofilia sono le neoplasie, in particolare quelle ematologiche, le malattie reumatiche come l'artrite reumatoide, la fascite eosinofila, le vasculiti, alcune patologie del tratto gastroenterico, dell'apparato respiratorio e le infezioni da HTLV (Human T-Lymphotropic Virus).

L'ipereosinofilia deve essere distinta dalle sindromi ipereosinofile (HES), che costituiscono un raro ed eterogeneo gruppo di disordini caratterizzati da persistente e marcata ipereosinofilia $\left(>1,5 \times 10^{9} / \mathrm{L}\right.$ per più di 6 mesi consecutivi) associati a evidenza di danno d'organo indotto dalle cellule eosinofile, laddove siano escluse altre cause. La prevalenza di queste forme è sconosciuta e insorgono più frequentemente nell'età giovane adulta con una predominanza tra $i$ maschi [2].

Il danno agli organi bersaglio mediato dagli eosinofili è molto variabile; in oltre il $50 \%$ dei casi possono essere coinvolti la cute, il cuore, i polmoni, il sistema nervoso centrale e periferico; inoltre, si possono avere disturbi della coagulazione.

Nel caso di ipereosinofilia secondaria, la terapia deve essere mirata a eradicare, quando possibile, la causa e allo stesso tempo al controllo delle complicanze dovute all'infiltrazione dei tessuti e al rilascio del contenuto dei granuli. In particolare l'elemento distintivo dei granuli eosinofili è il core cristallino costituito da una proteina ricca di arginina, la proteina basica maggiore, ad attività istaminasica e importante nella difesa dell'ospite contro i parassiti. Nella matrice dei granuli si trovano, inoltre, una perossidasi che catalizza l'ossidazione di molte sostanze e facilita il killing dei microrganismi, la proteina cationica eosinofila e la neurotossina, due ribonucleasi importanti nella difesa da agenti virali.

Per quanto riguarda le HES, la presa in carico terapeutica dipende dalla gravità della malattia e dal tipo di variante patogenetica. I corticosteroidi e l'idrossiurea sono le pietre miliari del trattamento, mentre l'imatinib rappresenta la terapia di prima scelta per i pazienti con ipereosinofilia clonale. Tale patologia è dovuta a delezione interstiziale del cromosoma $4 \mathrm{q} 12$ che causa la fusione di due geni FIP1L1-PDGFRA (F/P); il gene di fusione codifica una proteina
Tabella 1 Classificazione delle eosinofilie polmonari.

\begin{tabular}{cl}
\hline Eosinofilie & - Limitate al polmone \\
polmonari & Polmonite eosinofila semplice o \\
di origine & sindrome di Loeffler \\
sconosciuta & Polmonite eosinofila cronica \\
& Polmonite eosinofila acuta \\
& - In sindromi sistemiche \\
& Sindrome di Churg-Strauss \\
& Sindrome ipereosinofila idiopatica \\
& DRESS (Drug Rush with Eosinophilia \\
& and Systemic Symptoms) syndrome \\
Eosinofilie & - Eosinofilie polmonari di origine \\
polmonari di & parassitaria \\
origine nota & - Eosinofilie polmonari da altre \\
& cause infettive \\
& - Eosinofilie polmonari indotte da farmaci \\
& - Aspergillosi broncopolmonare allergica \\
\hline
\end{tabular}

con attività tirosinchinasica costitutiva. Recenti osservazioni suggeriscono che il mepolizumab, un anticorpo anti-inteleuchina 5, rappresenti una valida opzione terapeutica nei pazienti con sindrome ipereosinofila idiopatica che non sono portatori del gene di fusione F/P [2].

A livello dell'apparato respiratorio le eosinofilie polmonari (EP) rappresentano una varietà di patologie che si caratterizzano per l'accumulo patologico di eosinofili nelle vie aeree distali, negli spazi aerei e nel compartimento interstiziale del polmone. Possono associarsi a eosinofilia ematica e/o nell'espettorato.

Attualmente non esiste una classificazione delle EP universalmente accettata. La più conveniente dal punto di vista clinico distingue le forme idiopatiche limitate al polmone, o inquadrate in sindromi sistemiche, da quelle a eziologia nota (tabella 1) [3].

Indipendentemente dall'origine, tali affezioni possono differenziarsi sul piano clinico-radiologico, tanto da poter essere distinte in: sindrome di Loeffler (polmonite eosinofila semplice), polmonite eosinofila cronica e polmonite eosinofila acuta.

\section{Sindrome di Loeffler}

La sindrome di Loeffler (tabella 2) si caratterizza per una sintomatologia scarsa (o del tutto assente) che si associa alla presenza di infiltrati polmonari fugaci, migranti e spesso periferici, evidenti alla radiografia del torace come opacità unilaterali o bilaterali e a modesta eosinofilia ematica [4]. Numerose sono le cause che possono determinare tale sindrome: agenti infettivi e numerosi parassiti (causa più comune), farmaci, inalazione di pollini o sostanze vegetali;

Tabella 2 Sindrome di Loeffler.

- Sintomatologia clinica scarsa o del tutto assente

- Infiltrati polmonari fugaci (durata $<1$ mese)

- Eosinofilia ematica

- Eziologia varia (idiopatica in un terzo dei casi)

- Eccellente prognosi 
meno frequentemente neoplasie polmonari, asma, fibrosi polmonare idiopatica, sarcoidosi; in un terzo dei casi, tuttavia, non è possibile l'identificazione di una precisa causa [5]. Presenta eccellente prognosi con risoluzione spontanea, entro un mese, degli infiltrati polmonari e dell'eosinofilia ematica. Raramente necessita di terapia steroidea.

\section{Polmonite eosinofila cronica idiopatica}

La polmonite eosinofila cronica idiopatica (ICEP) (tabella 3) si caratterizza per un'insorgenza lenta e subdola, con sintomi respiratori quali tosse perlopiù non produttiva (nel $90 \%$ dei casi) e dispnea (50-90\% dei casi) [6] solitamente modesta e sintomi sistemici importanti quali febbre (in oltre il $60 \%$ dei casi) e dimagramento (fino al 75\% dei casi) [6]. La radiografia del torace mostra, nei due terzi dei casi, un quadro patognomonico detto a "fotografia negativa dell'edema polmonare" [7] contrassegnato da opacità sfumate, disomogenee, a densità variabile, non segmentarie e a prevalente localizzazione periferica con distribuzione a livello degli apici e delle regioni ascellari; poco frequenti sono versamenti pleurici e incremento di volume dei linfonodi mediastinici. La tomografia computerizzata (TC) del torace aggiunge, rispetto alla radiografia standard, l'evidenza di aree e/o chiazze di attenuazione tipo "vetro smerigliato" e di linfonodi mediastinici ingranditi.

Frequenti sono l'eosinofilia ematica (eosinofili $\geq 1 \times 10^{9} /$ L) [6] e incrementi della velocità di eritrosedimentazione e della proteina C-reattiva; nel $50 \%$ dei casi si ha un aumento delle IgE sieriche, che rispecchia l'alta percentuale di pazienti atopici.

In assenza di aumentata eosinofilia ematica la diagnosi viene posta tramite la dimostrazione di alveolite eosinofila (eosinofili $>40 \%$ ) al lavaggio bronchiolo-alveolare (BAL), principale procedura diagnostica che quasi sempre evita l'esecuzione della biopsia polmonare.

La terapia (tabella 4), basata sull'assunzione di steroidi orali per un periodo compreso tra 6 e 12 mesi, determina in poche ore la normalizzazione dei sintomi e in pochi giorni dei rilievi laboratoristici e radiologici [6]. Tale "drammatica risposta alla terapia" fa del trattamento steroideo un "test diagnostico" per ICEP [8].

Le ricadute, alla sospensione del trattamento o alla riduzione dei dosaggi, sono frequenti: si osservano in quasi la metà dei pazienti [6] e comunque rispondono ottimamente alla ripresa del trattamento steroideo.

Basandosi sull'osservazione della bassa percentuale di recidive nei pazienti asmatici in trattamento cronico con

Tabella 3 Polmonite eosinofila cronica idiopatica.

- Insorgenza lenta e subdola

Sintomi respiratori presenti da almeno 1 mese prima della diagnosi

- Infiltrati polmonari bilaterali alla radiografia del torace con prevalente distribuzione periferica

- Eosinofilia alveolare e/o ematica

Lavaggio bronchiolo-alveolare: eosinofili $>40 \%$

Eosinofilia ematica $\geq 1.000 / \mathrm{mm}^{3}$

- Asma presente nel 50\% dei casi

- Esclusione di cause note di patologie eosinofile polmonari
Tabella 4 Polmonite eosinofila cronica idiopatica: terapia.

- Risposta "drammatica" alla terapia steroidea della sintomatologia, dell'eosinofilia ematica e degli infiltrati radiologici

- Durata del trattamento: 6-12 mesi

- Frequenti recidive alla riduzione del trattamento o alla sua sospensione

- Ruolo preventivo sulle recidive dei corticosteroidi per via inalatoria

Tabella 5 Polmonite eosinofila cronica idiopatica: diagnosi differenziale.

- Polmonite eosinofila acuta idiopatica

Sintomatologia a insorgenza più rapida e importante

Assenza di atopia

Ipossiemia importante fino alla ventilazione meccanica non invasiva

Infiltrati polmonari senza prevalente distribuzione periferica

Eosinofilia ematica non caratteristica

Miglioramento "drammatico" con o senza terapia steroidea

Assenza di recidive

- Eosinofilie polmonari a causa nota

- Aspergillosi broncopolmonare allergica (APBA)

- Sindrome di Churg-Strauss

- Polmonite organizzativa criptogenetica

- Neoplasie e infezioni

steroidi per via inalatoria affetti da ICEP [9], al fine di ridurre le recidive, è stato proposto con successo l'utilizzo della terapia steroidea per via inalatoria [10].

Le patologie che entrano in diagnosi differenziale con la polmonite eosinofila cronica sono riportate nella tabella 5 .

\section{Polmonite eosinofila acuta idiopatica}

La polmonite eosinofila acuta idiopatica (IAEP) differisce dalla forma cronica per la modalità di insorgenza, la gravità della sintomatologia, l'assenza di atopia, la differente distribuzione degli infiltrati polmonari alla radiografia del torace, la remissione completa anche in mancanza di trattamento e l'assenza di recidive nei pazienti trattati.

Fondamentale è l'esclusione di tutte quelle forme di EP a causa nota, quali:

- le forme dovute a farmaci, in particolare gli antinfiammatori non steroidei, inclusi i salicilati, di largo utilizzo nelle patologie infiammatorie intestinali (per esempio, sulfasalazina), e gli antibiotici. Fondamentale è in questi casi un'approfondita anamnesi farmacologica. Utile può essere la consultazione del sito www.pneumotox.com [11], che fornisce informazioni sugli effetti a livello polmonare dei farmaci;

- le forme secondarie a infezioni parassitarie. In proposito, occorre ricordare che nelle aree non tropicali i principali agenti eziologici sono Ascaris lumbricoides, Toxocara canis, 
Taenia saginata, Trichinella spiralis e Fasciola hepatica, che si associano a eosinofilia ematica e infiltrati polmonari. Per la diagnosi si consiglia l'uso dei test microbiologici sul siero e sulle feci;

- l'aspergillosi broncopolmonare allergica (ABPA), sindrome clinica che si manifesta in soggetti affetti da asma atopico cronico che sviluppano ipersensibilità ad Aspergillus fumigatus, caratterizzata da infiltrati polmonari migranti, eosinofilia ematica e polmonare, bronchiectasie tipicamente centrali, aumentati tassi di IgE sieriche, presenza di precipitine sieriche specifiche per $A$. fumigatus [12].

Normali tassi di IgE totali escludono l'eziologia parassitaria e l'ABPA.

La sindrome di Churg-Strauss è una patologia sistemica caratterizzata da asma, infiltrati polmonari fugaci, ipereosinofilia e vasculite eosinofila sistemica; differisce dalla ICEP, di cui in alcuni casi rappresenta un continuum, per il coinvolgimento di altri organi (cuore, cute, tratto gastroenterico, sistema nervoso) oltre al polmone [13].

La polmonite organizzativa criptogenetica mostra similitudini dal punto di vista clinico e radiologico con la ICEP, ma non si associa a eosinofilia ematica; presenta alveolite linfocitaria al BAL e le lesioni polmonari predominano ai lobi inferiori [14].

La IAEP (tabella 6) si caratterizza per l'insorgenza di malattia acuta febbrile della durata da 1 a 7 giorni che si associa a dispnea, tosse, dolore toracico di tipo pleuritico, mialgie. La dispnea può essere molto importante, con ipossiemia grave, tale da richiedere l'uso della ventilazione meccanica in un'alta percentuale di pazienti. Radiologicamente (tabella 7) la IAEP si caratterizza per la comparsa di

Tabella 6 Polmonite eosinofila acuta idiopatica.

- Insorgenza acuta febbrile

- Sintomi respiratori presenti da meno di 7 giorni dalla diagnosi

- Grave ipossiemia: $\mathrm{PaO}_{2}$ in aria ambiente $(<60 \mathrm{mmHg})$

- Radiografia del torace: infiltrati polmonari bilaterali senza prevalente distribuzione periferica

- Eosinofilia alveolare

Lavaggio bronchiolo-alveolare: eosinofili $>25 \%$

Biopsia: polmonite eosinofila

- Assenza di atopia

- Esclusione di cause note di patologie polmonari eosinofile

Tabella 7 Polmonite eosinofila acuta idiopatica: radiologia.

- Radiografia del torace

Lesioni iniziali caratterizzate da interessamento interstiziale (strie di Kerley di tipo B)

A distanza di ore/giorni comparsa di esteso impegno alveolare o misto alveolare-interstiziale (maggiore alle basi)

- TC del torace

Infiltrati alveolari parenchimali diffusi

Aree di attenuazione tipo "vetro smerigliato"

Versamento pleurico

Rara la localizzazione periferica
Tabella 8 Polmonite eosinofila acuta idiopatica: terapia.

- Pronta e completa risposta ai corticosteroidi (casi di risposta in assenza di trattamento)

- Assenza di ricadute alla sospensione della terapia

Tabella 9 Polmonite eosinofila acuta idiopatica: diagnosi differenziale.

- Polmonite eosinofila cronica

Sintomatologia a insorgenza lenta e subdola

Atopia nel $50 \%$ dei casi

Infiltrati polmonari con prevalente distribuzione periferica

Caratteristica l'eosinofilia ematica e/o alveolare

Miglioramento "drammatico" dopo terapia steroidea

Frequenti recidive

- Polmonite eosinofila a causa nota

- Sindrome di Churg-Strauss

Vasculite sistemica

Si associa ad asma

Caratteristica l'eosinofilia ematica

Coinvolgimento multiorgano

- ALI/ARDS (Acute Lung Injury/Acute Respiratory Distress Syndrome)

infiltrati bilaterali diffusi con risparmio della periferia (quadro "simil-edema polmonare acuto"). Le lesioni, inizialmente di tipo interstiziale, a distanza di ore-giorni evolvono determinando un esteso impegno alveolare (da noduli ad addensamenti) o misto alveolare-interstiziale che tende a localizzarsi a livello delle basi polmonari. La TC evidenzia aree di attenuazione a "vetro smerigliato" e versamento pleurico mono o bilaterale, presente nel corso della malattia in tutti i pazienti. L'aumento degli eosinofili nel sangue non è caratteristico, benché sia possibile un loro transitorio incremento nel $50 \%$ dei pazienti. Al contrario, in modo caratteristico, la percentuale di eosinofili nel BAL appare elevata ( $>25 \%$ ). Anche per la IAEP il riscontro di alveolite eosinofila al BAL può evitare l'esecuzione della biopsia polmonare, che rimane comunque indicata nei soggetti immunodepressi.

La terapia steroidea ad alti dosaggi (tabella 8) determina una rapida risposta entro i primi giorni senza presentare, contrariamente ai paziente affetti da ICEP, recidive alla sospensione del trattamento. In letteratura, inoltre, diversamente che nella ICEP, sono stati segnalati casi di guarigione spontanea $[15,16]$. Oltre alla terapia steroidea alcuni pazienti richiedono ventilazione meccanica, che determina un rapido miglioramento della dispnea e dell'ossiemia [15].

Le patologie che entrano in diagnosi differenziale con la polmonite eosinofila acuta sono riportate nella tabella 9 [17-19].

\section{Caso clinico}

Donna, 76 anni, ex fumatrice, viene ricoverata presso l'UOC di Medicina Interna del Nuovo Ospedale Civile di Sassuolo (MO) per incremento della dolorabilità a livello lombosacrale, esacerbata dai movimenti e in assenza di traumi. Tale sintomatologia era stata la causa di un recente ricovero 
presso la medesima UOC. In quell'occasione la paziente era stata dimessa con diagnosi di "crolli vertebrali multipli" secondari a osteoporosi, con il consiglio di una terapia calcio-vitaminica e antiriassorbitiva con bifosfonati da associarsi all'uso di un appropriato busto ortopedico.

All'ingresso in reparto la paziente appare di umore deflesso e sofferente; si evoca vivo dolore in sede lombare alla digitopressione della muscolatura paravertebrale. Il restante esame obiettivo risulta negativo, così come negativi sono gli esami bioumorali e la radiografia del torace.

Alla terapia in essere vengono associati un antidolorifico (tramadolo cloridrato) e un antidepressivo (sertralina) con una buona risposta clinica, tale da indurre a considerare la paziente dimissibile; in quinta giornata dall'ingresso in reparto, però, la paziente manifesta dispnea per minimi sforzi, associata alla comparsa di crepitazioni bibasali teleinspiratorie "a velcro" in assenza di edemi declivi. A tali segni e sintomi si somma la comparsa di insistente tosse secca e di febbre.

Nel sospetto di un episodio infettivo polmonare, viene iniziato un trattamento antibiotico empirico con ceftriaxone al dosaggio di $2 \mathrm{~g} /$ die ev e viene richiesta una radiografia del torace, che mostra un quadro di congestione vascolare e impegno interstiziale. Due giorni dopo si assiste alla comparsa di una manifestazione cutanea, interpretata come angioite tossico-allergica. Viene quindi sospesa la terapia antibiotica in atto e iniziato un trattamento con levofloxacina, oltre all'utilizzo di cortisonici e antistaminici, con rapida regressione del quadro cutaneo. Per la persistenza della febbre, della tosse e l'aggravamento della dispnea, documentata dal quadro emogasanalitico che mostrava un'importante insufficienza respiratoria $\left(\mathrm{pO}_{2}=46 \mathrm{mmHg}\right.$ in aria ambiente), in decima giornata viene eseguita TC ad alta risoluzione (HRTC) del torace che evidenzia, bilateralmente a livello dorsale, aree consolidative compatte con broncogramma aereo e voluminose adenomegalie in sede ilare e sottocarenale (fig. 1). Alla luce di tale reperto TC associato ai sintomi e segni prima riportati e all'osservazione di un graduale aumento dell'eosinofilia ematica, passata da $0,41 \times 10^{9} / \mathrm{L}(6 \%)$ in seconda giornata a $7,56 \times 10^{9} / \mathrm{L}(35 \%)$ in tredicesima, seguendo il protocollo

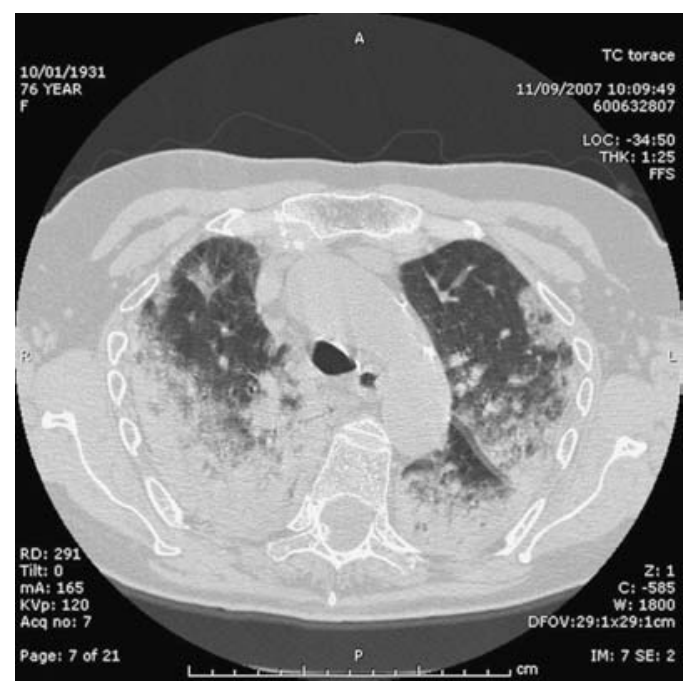

Figura 1 TC ad alta risoluzione del torace: presenza, bilateralmente a livello dorsale, di aree consolidative compattecon broncogramma aereo.

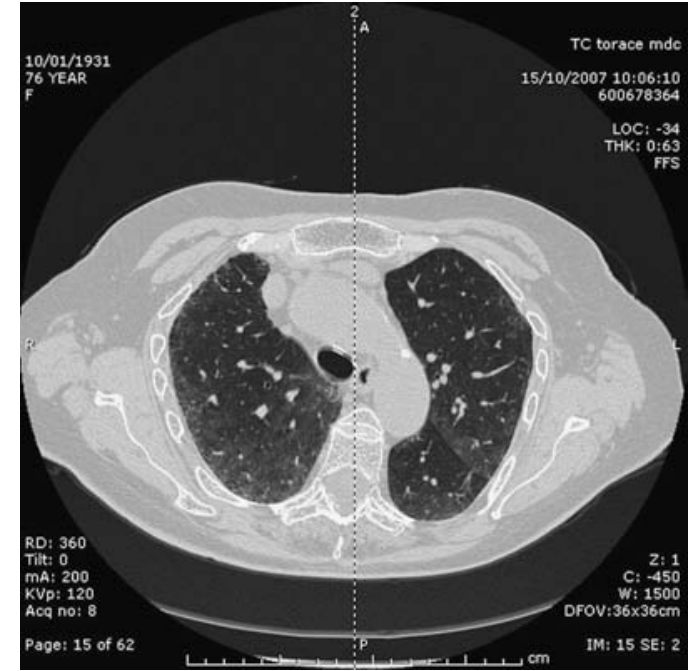

Figura 2 TC ad alta risoluzione del torace dopo un mese diterapia: scomparsa pressoché totale degli addensamenti parenchimali precedentemente descritti.

operativo delle insufficienze respiratorie acute del Dipartimento del nostro Ospedale, la paziente viene trasferita presso I'UOC di Pneumologia con la diagnosi di insufficienza respiratoria acuta ipossiemica da polmonite eosinofila acuta (da farmaci?).

All'ingresso in reparto, nel sospetto che la causa potesse essere secondaria a farmaci (sertralina), si sospende immediatamente la terapia antidepressiva e si inizia terapia steroidea con metilprednisolone ad alte dosi (1,5 mg/ $\mathrm{kg} / \mathrm{die})$; la terapia antibiotica precedentemente impostata viene mantenuta e di aggiunge terapia antifungina con itraconazolo in attesa delle indagini sierologiche e microbiologiche su espettorato, sangue e feci per la ricerca di germi comuni e parassiti, risultate poi tutte negative. Non è stato possibile eseguire l'esame broncoscopico per la grave insufficienza respiratoria della paziente.

In diciassettesima giornata si assiste al completo sfebbramento, che però si associa a importante peggioramento dell'ossiemia $\left(\mathrm{pO}_{2}=39 \mathrm{mmHg}\right)$ nonostante gli alti flussi di ossigeno. Viene ripetuta la HRTC, che mostra una minima riventilazione degli addensamenti precedentemente segnalati (quadro sostanzialmente stabile).

Dalla giornata seguente si assiste a un rapido miglioramento dell'ossiemia, della sintomatologia e alla riduzione dell'eosinofilia ematica fino alla completa normalizzazione.

Alla dimissione, avvenuta in quarantesima giornata, alla paziente viene consigliato di proseguire la terapia steroidea con metilprednisolone al dosaggio di $32 \mathrm{mg} /$ die. A distanza di 2 settimane, la successiva rivalutazione clinica consente un'ulteriore riduzione della terapia steroidea. La HRTC di controllo a un mese dall'inizio della terapia steroidea (fig. 2) mostra la scomparsa pressoché totale degli addensamenti parenchimali precedentemente descritti.

\section{Discussione}

Nel caso specifico, la diagnosi di polmonite eosinofila da farmaci (sertralina) è stata supportata dall'insorgenza acuta 
del quadro clinico caratterizzato da tosse secca insistente, ipossiemia grave e febbre, dalla presenza di elevati livelli di eosinofilia ematica (anche se questa non rappresenta un elemento determinante per la diagnosi), dal quadro radiologico a interessamento inizialmente interstiziale e poi alveolare, dalla "drammatica" risposta al trattamento steroideo in termini clinici, laboratoristici e radiologici e dall'esclusione delle principali cause note di polmonite eosinifila acuta.

Nel nostro caso non è stata possibile la determinazione dell'eosinofilia alveolare, caratteristica della forma acuta, tramite lavaggio bronchiolo alveolare e biopsia transbronchiale (TBB), in quanto l'improvviso aggravamento della paziente ha richiesto la rapida introduzione del trattamento steroideo.

Consultando il sito www.pneumotox.com [11], periodicamente aggiornato, nel quale il nome del principio attivo di un farmaco è messo in relazione con il pattern radiologico corrispondente, abbiamo constatato che il caso da noi descritto (polmonite eosinofila acuta da sertralina) è il secondo in letteratura [20].

La polmonite eosinofila acuta da noi diagnosticata potrebbe essere considerata parte di una sindrome da ipersensibilità secondaria all'esposizione a un farmaco (DRESS syndrome: Drug Rush with Eosinophilia and Systemic Symptoms) caratterizzata da 1) rush cutaneo (angioite tossicoallergica nel nostro caso), 2) incremento dell'eosinofilia e 3) coinvolgimento sistemico (linfoadenopatie presenti alla prima TC del torace, polmonite interstiziale nel nostro caso); la presenza di tutti e tre i criteri conferma la diagnosi [21]. La patogenesi è dovuta a un meccanismo di ipersensibilità di tipo non immediato o a un difetto nella capacità detossificante dei prodotti metabolici tossici del farmaco $[21,22]$. Il test cutaneo più sicuro per dimostrare l'ipersensibilità non immediata al farmaco è il patch test, da eseguire tra le 6 settimane e i 6 mesi dalla remissione completa [23], periodo non ancora trascorso nel nostro caso specifico.

Questo caso clinico ci ha indotti ad approfondire la complessa tematica delle eosinofilie polmonari con una revisione della letteratura in grado di puntualizzarne gli aspetti classificativi, radiologici, clinici e terapeutici.

\section{Conflitto di interessi}

Gli autori dichiarano di essere esenti da conflitto di interessi.

\section{Bibliografia}

[1] Rothenberg ME. Eosinophilia. N Engl J Med 1998;338(22):1592600.

[2] Roufosse FE, Goldman M, Cogan E. Hypereosinophilic syndromes. Orphanet J Rare Dis 2007;2:37.

[3] Donner CF, Sanguinetti CM (Eds.), Trattato italiano di pneumologia. Vol II. T I. Pisa: Edi-Aipo Scientifica, 2000: pp. 1291308.
[4] Loeffler W. Zur differential-diagnose der lungen-infiltrierungen. Il Uber fluchtige succedan-infiltrate (mit eosinophilie). Eitr Klin Tuberk 1932;79:368-92.

[5] Ford RM. Transient pulmonary eosinophilia and asthma: a review of 20 cases occurring in 5,702 asthma sufferers. Am Rev Respire Dis 1966;93:797-803.

[6] Marchand E, Reynaud-Gaubert M, Lauque D, Durieu J, Tonnel $A B$, Cordier JF. Idiopathic chronic eosinophilic pneumonia. A clinical and follow-up study of 62 cases. The Groupe d'Études et de Réchèrche sur les Maladies "Orphelines" Pulmonaires (GERM"O"P). Medicine (Baltimore) 1998;77(5):299-312.

[7] Jederlinic PJ, Sicilian L, Gaensler EA. Chronic eosinophilic pneumonia. A report of 19 cases and a review of the literature. Medicine (Baltimore) 1988;67(3):154-62.

[8] Carrington CB, Addington WW, Goff AM, Madoff IM, Marks A, Schwaber JR, et al. Chronic eosinophilic pneumonia. N Engl J Med 1969;280(15):787-98.

[9] Marchand E, Etienne-Mastroianni B, Chanez P, Lauque D, Leclerc $P$, Cordier JF. Groupe d'Études et de Réchèrche sur les Maladies Orphelines Pulmonaires. Idiopathic chronic eosinophilic pneumonia and asthma: how do they influence each other? Eur Respir J 2003;22(1):8-13.

[10] Naughton M, Fahy J, Fitzgerald MX. Chronic eosinophilic pneumonia. A long-term follow-up of 12 patients. Chest 1993;103(1): $162-5$.

[11] Pneumotox on line. The drug-induced lung diseases. http:// www.pneumotox.com.

[12] Greenberger PA. Allergic bronchopulmonary aspergillosis and fungoses. Clin Chest Med 1988;9(4):599-608.

[13] Gross WL, Reinhold-Keller E. Churg-Strauss syndrome. Orphanet Encyclopedia, October 2002. http://www.orpha.net/data/ patho/Pro/en/ChurgStrauss-FRenPro745.pdf.

[14] Cordier JF. Organising pneumonia. Thorax 2000;55(4):318-28.

[15] Philit F, Etienne-Mastroianni B, Parrot A, Guerin C, Robert D, Cordier JF. Idiopathic acute eosinophilic pneumonia. A study of 22 patients. Am J Respir Crit Care Med 2001;163:A980.

[16] Hayakawa H, Sato A, Toyoshima M, Imokawa S, Taniguchi M. A clinical study of idiopathic eosinophilic pneumonia. Chest 1994;105(5):1462-6.

[17] Bernard GR, Artigas A, Brigham KL, Carlet J, Falke K, Hudson L, et al. The American-European Consensus Conference on ARDS. Definitions, mechanisms, relevant outcomes, and clinical trial coordination. Am J Respir Crit Care Med 1994;149(3 Pt 1):818-24.

[18] Steinberg KP, Hudson LD. Acute lung injury and acute respiratory distress syndrome. The clinical syndrome. Clin Chest Med 2000;21(3):401-17.

[19] Weiland JE, Davis WB, Holter JF, Mohammed JR, Dorinsky PM, Gadek JE. Lung neutrophils in the adult respiratory distress syndrome. Clinical and pathophysiologic significance. Am Rev Respir Dis 1986;133(2):218-25.

[20] Barnés MT, Bascuñana J, García B, Alvarez-Sala JL. Acute eosinophilic pneumonia associated with antidepressant agents. Pharm World Sci 1999;21(5):241-2.

[21] Vaillant L. Drug hypersensitivity syndome: drug rush with eosinophilia and systemic symptoms (DRESS). J Dermatol Treat 1999;10:267-72.

[22] Sullivan JR, Shear NH. The drug hypersensitivity syndrome: what is the pathogenesis? Arch Dermatol 2001;137(3):357-64.

[23] Barbaud A, Gonçalo M, Bruynzeel D, Bircher A, European Society of Contact Dermatitis. Guidelines for performing skin tests with drugs in the investigation of cutaneous adverse drug reactions. Contact Dermatitis 2001;45(6):321-8. 